\title{
Patrones de las enfermedades raras congénitas en pacientes ingresados en un hospital regional
}

\author{
Patterns of rare congenital diseases in patients admitted \\ to a regional hospital
}

\author{
M.C. Martínez Cirre ${ }^{1}$, M. M. Rodríguez del Águila ${ }^{2}$, A. Fernández Valdivia ${ }^{1}$, \\ M. Peña Taveras ${ }^{1}$, J. Martínez Tapias ${ }^{1}$
}

\section{RESUMEN}

Fundamento. Describir el patrón de pacientes ingresados por enfermedades raras correspondientes a anomalías congénitas en un hospital regional.

Métodos. Estudio transversal retrospectivo. Se consideraron las altas de hospitalización de los años 20092012 con diagnóstico principal entre los códigos CIE 9R MC 740-759. La fuente de información fue el Conjunto Mínimo Básico de Datos. Se analizaron variables sociodemográficas y clínicas.

Resultados. Ingresan en el hospital por enfermedades raras congénitas un 1,6\% de la población Un 58,5\% fueron varones, con edad media de 21,4 \pm 21,5 años. Las Categorías Diagnósticas Mayores más frecuentes fueron Enfermedades del sistema nervioso (86,9\%), Enfermedades del aparato circulatorio (51,7\%) y Sistema músculo-esquelético (50,3\%). El 18\% de las hospitalizaciones correspondieron a reingresos de pacientes. El servicio con mayor número de episodios fue Cirugía Pediátrica, 29\%, seguido de Neurocirugía, 20\%.

Conclusiones. El patrón de enfermedad rara congénita en el hospital Universitario Virgen de las Nieves corresponde a un paciente joven, con una enfermedad perteneciente al grupo de la Categoría Diagnóstica Mayor Enfermedad del sistema nervioso tratada quirúrgicamente y un porcentaje de reingresos no elevado.

Palabras clave. Enfermedades raras. Anomalías congénitas. Conjunto Mínimo Básico de Datos. Asistencia hospitalaria

\begin{abstract}
Background. To describe the pattern of patients admitted due to rare diseases corresponding to congenital anomalies in a regional hospital.

Methods. Retrospective transversal study. We considered hospital discharges for the years 2009-2012 with principal diagnosis between codes CIE 9R MC 740-759. The source of information was the Basic Minimum Data Set. Socio-demographic and clinical variables were analyzed.
\end{abstract}

Results. One point six percent (1.6\%) of the population was admitted to hospital due to rare congenital diseases. Fifty-eight point five percent (58.5\%) were male, with average age $21.4 \pm 21.5$ years. The major diagnostic categories were: diseases of the nervous system (86.9\%), circulatory systems diseases $(51.7 \%)$ and musculoskeletal system diseases (50.3\%). Eighteen percent (18\%) of hospital admissions corresponded to patient readmissions. The service with the greatest number of episodes was Pediatric Surgery, $29 \%$, followed by Neurosurgery, $20 \%$.

Conclusions. The pattern of rare congenital disease in the "Virgen de Nieves" University Hospital corresponds to a young patient, with a disease belonging to the diseases of the nervous system group of the major diagnostic categories, treated surgically, and with a low percentage of readmissions.

Key words. Rare disease. Congenital abnormalities. Set minimum basic of clinical data. Hospital care.
1. Servicio de Documentación Clínica y Archivo. Hospital Virgen de las Nieves. Granada.

2. UGC Medicina Preventiva, Vigilancia y Promoción de la Salud

Recepción: 15 de enero de 2013

Aceptación provisional: 26 de febrero de 2013

Aceptación definitiva: 3 de abril de 2013

\section{Correspondencia:}

$\mathrm{M}^{\mathrm{a}}$ Carmen Martínez Cirre

Servicio de Documentación Clínica y Archivo

Hospital Virgen de las Nieves

Avda. Fuerzas Armadas, 2

18014 Granada (Spain)

E-mail: carmen.martinez.cirre.sspa@

juntadeandalucia.es 


\section{INTRODUCCIÓN}

El concepto de Enfermedad Rara (ER) se pone en circulación por primera vez a mediados de los años 80 en Estados Unidos y siempre estrechamente relacionado con el concepto de medicamentos huérfanos. Ambos términos se desarrollan en paralelo y se dirigen a dar solución a los problemas que tienen las enfermedades de baja prevalencia. Engloban a un grupo de enfermedades muy diversas, la mayoría de ellas con base genética y baja prevalencia, aunque no debemos excluir el componente ambiental. Por lo general, tienen una evolución crónica y muy severa, con múltiples deficiencias motoras, sensoriales y cognitivas, por lo que suelen presentar un alto nivel de complejidad clínica que dificulta su diagnóstico y tratamiento ${ }^{1}$.

Se define como ER aquella entidad clínica cuya prevalencia no supera la cifra de 5 casos por cada 10.000 habitantes en la Comunidad Europea. La mayoría de los casos de ER aparecen en la edad pediátrica; esto es debido a la alta frecuencia de las de origen genético. Sin embargo, la mayor prevalencia en la edad adulta se debe, fundamentalmente, a un elevado índice de mortalidad en las enfermedades infantiles y a las diagnosticadas de forma tardía y no demasiado severas ${ }^{2}$.

Se calcula que existen, aproximadamente, entre 5.000 y 8.000 enfermedades que pueden ser catalogadas como ER que afectan al 6 u $8 \%$ de la población, es decir, entre 24 y 36 millones de personas; de los cuales tres millones, residen en España ${ }^{1}$.

En la actualidad las ER se clasifican en congénitas y no congénitas, correspondiendo la gran mayoría al primer grupo. Las últimas estimaciones acerca del porcentaje de enfermedades genéticas incluidas bajo esta definición informan de que al menos un $75 \%$ de las ER tendrían este origen $^{3}$. Sin embargo, esta cifra hay que manejarla con cuidado, porque dada la eclosión de nuevos hallazgos y variantes genéticas, no es extraño que se ofrezcan cifras de hasta 8.000 enfermedades diferentes y que de forma grosera se pueda hablar de que un $6 \%$ de la población total de la UE estaría afectada en algún momento de su vida por una de estas enfermedades ${ }^{4}$.
La investigación de las ER requiere la colaboración entre equipos de diversas disciplinas y el acceso a datos y material biológico recogido en toda la UE, para alcanzar un tamaño adecuado de la muestra. Los proyectos de investigación conjunta y de coordinación son de particular interés en este ámbito, como también crear infraestructuras compartidas: registros, bases de datos, depósitos y plataformas técnicas ${ }^{5}$.

En base a estos antecedentes, el objetivo de este estudio fue realizar una descripción transversal retrospectiva de la enfermedades raras hospitalizadas que pertenecen al grupo de Anomalías Congénitas clasificadas en la Clasificación Internacional de Enfermedades, 9 $9^{\text {a }}$ Revisión Modificación Clínica ${ }^{6}$ (CIE 9R MC) entre los códigos 740-759 en el Hospital Virgen de las Nieves de Granada desde el año 2009 hasta el primer semestre del año 2012.

\section{MATERIAL Y MÉTODOS}

Para la consecución del objetivo se llevó a cabo un estudio transversal retrospectivo. La población de estudio estuvo formada por las altas de hospitalización producidas en el hospital Virgen de las Nieves de Granada, situado entre los grandes hospitales del Sistema Sanitario Público de Andalucía, con un área de influencia en las provincias de Granada, Jaén y Almería y con una población de referencia de 442.523 habitantes. Está dotado de una cartera de servicios de alta complejidad y destaca por la incorporación a sus servicios de tecnologías de última generación. El estudio se realizó durante los años 2009-2011 y primer semestre de 2012. Como criterio de inclusión se tomó todos los episodios de hospitalización cuyo diagnóstico principal fuera alguna de las enfermedades raras congénitas que figuran en el listado de enfermedades raras de la Consejería de Salud de la Junta de Andalucía ${ }^{7}$ y cuyo código según la Clasificación Internacional de Enfermedades CIE 9R MC estaba comprendido entre 740 y 759 y que corresponde a la clasificación de Anomalías congénitas. La fuente de información considerada fue el Conjunto Mínimo Básico de Datos al alta (CMBD). 
El diagnóstico principal contenido en esta base de datos se obtuvo fundamentalmente del informe de alta, hoja operatoria e informe de anatomía patológica de la historia clínica, y fue codificado según la CIE 9R MC. Se realizó una agrupación de las altas hospitalarias por el programa clinos info mediante el All Patient GRD $27^{8}$.

Las variables analizadas fueron: edad del paciente, sexo, provincia de residencia, circunstancia de admisión (urgente/programado), motivo de alta (solo se analizó el exitus), días de estancia, clasificación y diagnóstico principal según código CIE 9R MC, y Categoría Diagnóstica Mayor (CDM). Existen 25 grandes capítulos o categorías diagnósticas mayores que se construyen a través de la agrupación de las altas hospitalarias mediante el sistema anteriormente descrito y que se forman siguiendo un algoritmo que tiene en cuenta como primera secuencia el diagnóstico principal ${ }^{9}$, muchas de ellas se ajustan a los grandes aparatos o sistemas corporales. Reingreso del paciente en el periodo analizado (sí/no) y servicio de alta. La variable dependiente fueron los días de estancia y como variables independientes se tomaron las restantes. La CDM 15 contiene todas las enfermedades congénitas correspondientes a niños con edad inferior a 28 días en el momento del ingreso.

Se realizó un análisis de pacientes y otro de episodios de hospitalización. Los pacientes que causaron varias altas se contabilizaron una única vez. Los datos se resumieron en torno a frecuencias y porcentajes para las variables cualitativas y medias y desviaciones para las variables cuantitativas. Se aplicó el test $\mathrm{t}$ de Student y Análisis de la Varianza para la comparación de medias.
Con las altas hospitalarias que cumplían las condiciones de nuestro estudio, se procedió a la disociación de la información confidencial del paciente, cumpliendo así con la Ley Orgánica 15/1999.

\section{RESULTADOS}

Durante el periodo analizado se hospitalizaron 1.534 pacientes con diagnóstico principal de ER clasificadas como anomalías congénitas, causando 1.866 altas hospitalarias (promedio de 1,2 episodios/ paciente). El porcentaje de estos episodios supone $1,6 \%$ del total del hospital.

La edad media de estos pacientes excluyendo la patología perinatal era de 21,4 \pm 21,5 años. Un $58,5 \%$ del total fueron varones. El 78\% ingresaron de forma programada y un $1 \%$ tuvieron como motivo de alta el fallecimiento. Un $73 \%$ de los episodios proceden de la provincia de Granada, le siguen Jaén y Almería con un 10\% y 8\%. La Categoría Diagnóstica Mayor (CDM) más frecuente fue la de Enfermedades del sistema nervioso (86\%), seguida de las Enfermedades del aparato circulatorio con un $51,7 \%$ y las Enfermedades del sistema músculo-esquelético perinatales con un $50,3 \%$.

El 18\% de las altas hospitalarias correspondieron a reingresos de pacientes; el servicio con mayor número de altas fue Cirugía Pediátrica con un $29 \%$, seguido del servicio de Neurocirugía, alcanzando un $20 \%$.

Analizando la distribución según CDM por sexo y edad (Tabla 1) se observan porcentajes similares en mujeres y varones excepto para la categoría 03 , donde hay una menor frecuencia de mujeres $(39,6 \%)$.

Tabla 1. Distribución de pacientes con anomalías congénitas por edad y sexo en las cinco categorías diagnósticas mayores más frecuentes

\begin{tabular}{lcccc}
\hline \multicolumn{1}{c}{ Categoría Diagnóstica Mayor } & Edad en años* & \% Mujeres & \% Varones & n \\
\hline 01 Sistema nervioso & $39,3 \pm 19,7$ & 50,7 & 49,3 & 274 \\
\hline 03 Oído, nariz, boca y faringe & $13,6 \pm 16,0$ & 39,6 & 60,4 & 192 \\
\hline 05 Aparato circulatorio & $24,7 \pm 22,6$ & 47,3 & 52,7 & 292 \\
\hline 08 Sistema músculo-esquelético & $10,3 \pm 13,9$ & 43,6 & 56,4 & 195 \\
\hline 15 Patología perinatal & $0,0 \pm 0,0^{* *}$ & 44,1 & 55,9 & 202 \\
\hline
\end{tabular}

*Media (desviación típica); ** recién nacidos con edad inferior a 28 días. 
Gran parte de las CDM agrupan los casos en torno a una clasificación mayor de códigos CIE 9R MC, siendo las más frecuentes: Anomalías congénitas del aparato circulatorio, para la categoría diagnóstica mayor 01 con un 86,9\%; Anomalías del bulbo arterioso y del cierre septal cardiaco, para la categoría 05 Aparato Circulatorio, con el $51,7 \%$; Ciertas deformidades músculo es- queléticas congénitas, para la categoría 08 Sistema músculo esquelético con el $50,3 \%$; Fisura del paladar y labio leporino para la categoría 03 Oído, nariz, boca y faringe, con un 44,8\% y Otras anomalías del aparato circulatorio y anomalías del aparato urinario, con un $12,4 \%$ y $11,4 \%$ respectivamente, para la categoría 15 Patología perinatal (Tabla 2).

Tabla 2. Categorías diagnósticas mayores más frecuentes en pacientes y su distribución según clasificación CIE 9R MC y diagnóstico más frecuente. Los porcentajes de cada celda se refieren al total de su grupo correspondiente

\begin{tabular}{|c|c|c|}
\hline $\begin{array}{c}\text { Categoría Diagnóstica } \\
\text { Mayor }\end{array}$ & $\begin{array}{l}\text { Clasificación CIE 9R MC } \\
\text { más frecuente (\%) }\end{array}$ & $\begin{array}{l}\text { Diagnóstico } \\
\text { más frecuente (\%) }\end{array}$ \\
\hline 01 Sistema nervioso & $\begin{array}{l}747 \text { Anomalías congénitas del ap. } \\
\text { circulatorio }(86,9 \%)\end{array}$ & $\begin{aligned} & 747.81 \text { Anomalías del sistema } \\
& \text { cerebrovascular }(85,4 \%)\end{aligned}$ \\
\hline $\begin{array}{l}03 \text { Oído, nariz, boca y } \\
\text { faringe }\end{array}$ & $\begin{array}{l}749 \text { Fisura del paladar y labio } \\
\text { leporino }(44,8 \%)\end{array}$ & $\begin{array}{l}749.20 \text { Fisura palatina junto labio } \\
\text { leporino }(20,8 \%)\end{array}$ \\
\hline 05 Ap. circulatorio & $\begin{array}{l}745 \text { Anomalías de bulbo arterioso } \\
\text { y del cierre septal cardiaco } \\
(51,7 \%)\end{array}$ & $\begin{array}{l}745.5 \text { Defecto del tabique } \\
\text { auricular tipo ostium } \\
\text { secundum }(37,0 \%)\end{array}$ \\
\hline $\begin{array}{l}08 \text { Sistema músculo- } \\
\text { esquelético }\end{array}$ & $\begin{array}{l}754 \text { Ciertas deformidades músculo- } \\
\text { esqueléticas congénitas (50,3\%) }\end{array}$ & 754.51 Talipes equinovaro $(13,8 \%)$ \\
\hline 15 Patología perinatal & $\begin{array}{l}747 \text { Otras anomalías del ap. } \\
\text { circulatorio (12,4\%) y } 753 \\
\text { anomalías del ap. urinario (11,4\%) }\end{array}$ & $\begin{array}{ll}747.0 & \text { Conducto arterioso } \\
& \text { permeable }(48,0 \%)\end{array}$ \\
\hline
\end{tabular}

Dentro de las anomalías congénitas del Aparato Circulatorio, que es la que más casos presenta, el diagnóstico que mas aparece corresponde a las Anomalías del sistema cerebrovascular con un $85,4 \%$ (Tabla 2). El diagnóstico más frecuente en la Patología Perinatal es la Estenosis pilórica hipertrófica congénita, con una diferencia evidente respecto a las mujeres $(87,8 \%$ frente a $12,2 \%$ ).

La estancia media para las ER congénitas es de 6,3 días, siendo la CDM de
Patología Perinatal la que tiene estancias mayores. Se observan diferencias significativas según la circunstancia de admisión en cuanto a los días de estancia, siendo la media superior cuando el ingreso fue urgente que programado $(15,2 \pm 24,1$ frente a $5,7 \pm 11,3, p<0,001)$. Por grupos de edad también se observan diferencias, siendo los menores de un año los que causan en promedio mayor número de estancias. No existen diferencias entre varones y mujeres ni según el año de alta (Tabla 3). 
Tabla 3. Relación de los días de estancia respecto a variables sociodemográficas y de admisión del paciente

\begin{tabular}{lccc}
\multicolumn{1}{c}{ Variable } & n & Media \pm desviación & p \\
\hline Sexo & & & \\
$\quad$ Varón & 698 & $7,8 \pm 17,1$ & ns \\
$\quad$ Mujer & 636 & $7,7 \pm 13,2$ & \\
\hline Grupos de edad & & & $<0,001$ \\
$\quad \leq 1$ año & 405 & $13,4 \pm 23,9$ & \\
$2-14$ años & 509 & $3,7 \pm 4,5$ & \\
15-40 años & 318 & $6,4 \pm 11,8$ & \\
$\quad \geq 41$ años & 302 & $8,7 \pm 14,9$ & \\
\hline Circunstancia de admisión & & & \\
Urgente & 339 & $15,2 \pm 24,1$ & \\
Programado & 1195 & $5,7 \pm 11,3$ & \\
\hline Año de alta & & & \\
2009 & 502 & $7,7 \pm 15,8$ & \\
2010 & 439 & $8,2 \pm 16,8$ & \\
2011 & 377 & $8,2 \pm 13,6$ & \\
2012 & 216 & $6,3 \pm 15,8$ & \\
\hline
\end{tabular}

ns: no significativo

\section{DISCUSIÓN}

Las anomalías congénitas más frecuentes durante el periodo comprendido entre 2009 y primer semestre de 2012 hospitalizadas por ese motivo en el hospital Virgen de las Nieves corresponden a anomalías en el sistema cardiovascular: corazón y sistema cerebrovascular. Aunque la categoría diagnóstica mayor con más casos corresponde a la 01 Enfermedades del sistema nervioso, el diagnóstico más frecuente dentro de esta categoría es la anomalía de los vasos cerebrovasculares (aneurismas o malformaciones arteriovenosas). Esto es totalmente plausible, ya que corazón y vasos sanguíneos tienen el mismo desarrollo embriológico.

No podemos descartar la inclusión de malformaciones vasculares cerebrales no congénitas en los resultados. Esto se debe a la poca especificidad que tiene el informe de alta sobre la etiología de esta patología. Es verdad que a veces el clínico tampoco puede asegurar definitivamente si son congénitas ${ }^{10}$. También hemos descartado en el estudio los episodios asistenciales cuyo diagnóstico principal es un aneurisma ce- rebral roto ${ }^{11}$; esta patología se clasifica con el diagnóstico principal de Hemorragia Subaracnoidea según las normas de codificación en CIE 9R MC y dicho código no está incluido en la lista de enfermedades congénitas; ello conlleva a que puede haber más aneurismas cerebrales congénitos de los que se presentan en este estudio. La alta prevalencia de este tipo de anomalías se da fundamentalmente en personas adultas. Ello puede ser debido al éxito del tratamiento en estas afecciones y a un elevado número de ingresos por paciente para control y seguimiento.

El defecto en el tabique auricular tipo Ostium Secundum es el segundo diagnóstico más frecuente en patologías congénitas que causan ingreso. En estos casos está clara la etiología congénita, puesto que la mayoría de estos pacientes lo hacen en la edad pediátrica ${ }^{12}$.

La categoría 15 Patología perinatal merece una mención especial ya que en ella se recogen cualquier anomalía congénita que se produce en neonatos, es decir, en niños ingresados que tienen una edad en días inferior o igual a 28 . Si el estudio se realiza de 
forma clasificatoria de diagnósticos, las más frecuentes serían las Anomalías del Aparato Circulatorio ${ }^{13}$, igual que la aparición en otras edades, o Anomalías en Aparato Urinario. $\mathrm{Si}$ el estudio lo realizamos por diagnóstico, el más frecuente es la Estenosis Pilórica Hipertrófica y con una clara diferencia de frecuencia, predominante en el sexo masculino, aunque esto es bien conocido en la etiopatogenia de esta anomalía ${ }^{14}$.

Es coherente que el servicio hospitalario con más ingresos sea el de Cirugía Pediátrica, puesto que la mayoría de las anomalías congénitas tiene como tratamiento la cirugía y las edades de los pacientes son fundamentalmente pediátricas. Al ser este hospital de tercer nivel y siendo el servicio de Neurocirugía de referencia para otras poblaciones distintas del área hospitalaria, es fácil entender que sea el segundo en ingresos hospitalarios.

La mortalidad producida en las anomalías congénitas clasificadas entre los códigos CIE 9R MC 740-759, es inferior a la media hospitalaria para este mismo periodo de tiempo (4,73\%). Llama la atención la baja mortalidad para este grupo de patologías, que podría ser debido a la efectividad en los tratamientos quirúrgicos y/o a la prevención antes del nacimiento (abortos).

Se ha observado que la estancia media por estas patologías es inferior a la media del hospital (7,97 días), no encontrando evidencias que justifiquen dicho resultado, exceptuando la edad del paciente (al ser pacientes más jóvenes, no son pluripatológicos).

Como conclusión se puede afirmar que el campo de las enfermedades raras es un área de investigación aún por explorar. Este estudio es pionero en la descripción de los patrones de enfermedades raras congénitas en pacientes hospitalizados. Se necesitan registros de información clínica de calidad en ER que confirmen los resultados obtenidos.

Las patologías analizadas corresponden a una parte de las anomalías congénitas, casi todas ellas con un tratamiento quirúrgico. Quedan por estudiar las enfermedades raras congénitas de tipo metabólico que están recogidas en otro apartado de la lista de ER, cuyo código CIE 9R MC está fuera del rango evaluado.

\section{BIBLIOGRAFÍA}

1. Salamanca C, Aldana J. Metodología para la elaboración del registro de enfermedades raras de Andalucía. Servicio Andaluz de Salud. Consejería de Salud, marzo 2012.

2. Posada M, Abata I. Recursos Asistenciales y de Investigación de Enfermedades Raras Ubicados en la Comunidad de Madrid. Promotor: Fundación TELETÓN FEDER para la Investigación de Enfermedades Raras. Estudio financiado por Merck España 2012.

3. Red Epidemiológica de Investigación de Enfermedades Raras (REpIER). http://www.isciii.es/ ISCIII/es/contenidos/fd-el-instituto/organizacion.shtml [acceso el 15 de diciembre de 2012].

4. Posada M, Martín-Arribas C, Ramírez A, Villaverde I, Enfermedades raras: Concepto, epidemiología y situación actual en España. An Sist Sanit Navar 2008; 31 (Supl. 2): 9-20.

5. Comisión Europea. Dirección General de Sanidad y Protección de los Consumidores. Dirección C-Salud pública y evaluación de riesgos C2 Información sobre la salud, 2008.

6. Ministerio de Sanidad, Política Social e Igualdad, Secretaría General Técnica. Clasificación Internacional de Enfermedades $9^{a}$ Revisión Modificación Clínica, $8^{\text {a }}$ edición, 2012.

7. Listado de anomalías congénitas. Consejería de Salud y Bienestar Social. Junta de Andalucía, http://www.juntadeandalucia.es/salud/ sites/csalud/contenidos/Informacion_General/ c_3_c_6_enfermedades_raras/listado_enfermedades [acceso 6 de octubre de 2012]

8. Iasist; SA Versión Andalucía. Suite Clinos versión 5.5 (Rev. 420) 2012.

9. САво J. Necesidad de nuevas formas y herramientas de gestión sanitaria. Gestión Sanitaria Integral: Pública y Privada, Centro de estudios financieros. $1^{\text {a }}$ edición, 2010.

10. JiMÉNEZ E. Aneurisma cerebral: ¿congénito o adquirido? Tribuna médica (Bogotá) 1999; 99: 29-42.

11. Cianfoni A, Pravatá E, De Blasi R, Tschour CS, BoNALDI G. Clinical presentation of cerebral aneurysms. Eur J Radiol. doi: 10.1016/j-ejrad. 2012. Epub ahead of print.

12. Sangeeta Priyadarshi S, Alpa Sanjeev A, Minakshi B. Prevalence, Pattern and Outcome of Congenital Heart Disease in Bhabha Atomic Research Centre Hospital, Mumbai. Indian J Pediatr. doi 10.1007/s12098-012-0910-x. Noviembre 2012.

13. Neha Trivedi, MD, Denis Levy, MD, Maryam Tarsa, MD et al, Congenital Cardiac Anomalies. Prenatal Readings Versus Neonatal Outcomes. J Ultrasound Med 2012; 31: 389-399.

14. Krogh C, Gørtz S, Wohlfahrt J, Biggar RJ, Melbye M, FIS CHER TK. Pre- and perinatal risk factors for pyloric stenosis and their influence on the male predominance. Am J Epidemiol 2012; 176: 24-31. 